\title{
An integrated low carbon energy solution to cooking fuel, tailored to Niger state's rural population
}

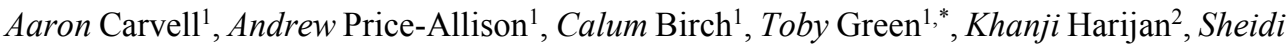 \\ Maihankuri $^{3}$, Abdulganiy Raji ${ }^{4}$, Mohammed Uqaili ${ }^{5}$, and Valerie Dupont ${ }^{1}$ \\ ${ }^{1}$ School of Chemical and Process Engineering, University of Leeds, Leeds, LS2 9JT, UK \\ ${ }^{2}$ Department of Mechanical Engineering, Mehran University of Engineering \& Technology, Jamshoro, \\ Pakistan \\ ${ }^{3}$ Mechanical Engineering, Petroleum Training Institute, Effurun, Delta State, Nigeria \\ ${ }^{4}$ Department of Agricultural and Environmental Engineering, University of Ibadan, Ibadan, Nigeria \\ ${ }^{5}$ Department of Electrical Engineering, Mehran University of Engineering \& Technology, Jamshoro, \\ Pakistan
}

\begin{abstract}
Niger State (Nigeria) was selected as a case study of renewable, affordable and user friendly clean energy provision in remote areas of developing countries. Niger state has $80 \%$ of its 4.5 million population living in rural agrarian areas with low literacy rates, there is a lack of wind thus eliminating wind as widely available potential power source. Based on the assessment of the local large insolation, the type of agricultural, biomass and husbandry resources, this study selected the design of anaerobic digestion units processing mostly animal and human waste, and whose heating and power requirement would be entirely provided by solar photovoltaic/thermal to maintain optimum efficiency of the biogas production. The designs was carried out at the scale of up to 15 household demand (community scale). Volume and therefore the production of biogas maybe increased or decreased in the design considered, and local, low cost resilient material were proposed. The proposed system was costed for a community of 24 people, demonstrating the potential for clean and renewable gas production economically.
\end{abstract}

\section{Introduction}

A range of influencing factors must be considered when choosing alternative low carbon energy solutions including; landscape, resource availability, resource and technology reliability and cost. Understanding the problems faced by these populations and of the nature and limits of their energy resources is required.

Nigeria is the most populous nation in Africa, the population is supported by a fertile landscape, a long rainy season with high annual rainfall and high solar irradiance throughout most of the nation. This large population is maintained despite an extremely low life expectancy, estimated at around 53 years [1]. Most of Nigeria's population live without

\footnotetext{
*Corresponding author: pmtag@leeds.ac.uk
} 
access to electricity, with electrification rates of around $40 \%$, and of those that do have access, the needs of only around $30 \%$ are adequately met, with frequent black-outs of varying length and timing. The power crisis that has been affecting Nigeria for many years now has been exacerbated by some key failings; poorly maintained/aging equipment and infrastructure, disruptions in supply of oil/gas and high level corruption [2]. In this aspect it is very similar to the current situation of Pakistan.

The energy usage figures for Nigeria provided by the International Energy Agency show the huge proportion of energy production coming from biomass, at $41.5 \%$ [3], however contrasted with the final energy consumption, for which biomass accounts for $86.2 \%$ [3], the existing importance of renewables in Nigeria can be seen.

Niger State, located in Western Nigeria, is the largest of Nigeria's 36 states, it accounts for almost $10 \%$ of the land area of the country, although its population is only 3.9 Million, $80 \%$ of which are rural dwellers [4] with literacy levels lower than the national average (37.5\%) [4], thus its economy is mostly based on agriculture, where "archaic" farming practices are still in use[5]. Eighty five percent of its land area is arable helped by long rainy season. The large rivers and their tributaries running through the state also give the opportunity for irrigation, not yet fully exploited [6]. It is rare for net electricity generation to exceed $50 \%$ of its capacity, the record high for 2016 being 5075 MW [7]. Niger State's large agricultural industry producing a lot of crop residue (from sugarcane, yam, cassava, onion, sweet potatoes) and animal wastes (poultry, cattle, goats, sheep), it benefits from good solar irradiance, adequate hydro potential but no significant wind power potential [8-10].

It is shown that consistent levels of solar radiation and sunny hours are received year long, with a slight decrease in August and increase in November for the heights of the wet and dry seasons respectively. This, along with this other data and literature has led the German Society for International Cooperation (GIZ) in partnership with the Nigerian government to highlight solar as a rapidly dispersible power generation source [8]. Numerous studies of varying depth and detail have looked at the hydro potential of Nigeria as a whole, an assessment of available data and literature showed that of a total 277 potential SHP sites 61 were located within Niger State. Throughout Nigeria, biomass is being burnt as a cooking fuel [9]. As detailed above, both solar and hydro are viable options for water heating (solar thermal) and power generation. Niger State was chosen as location for a case-study of integrated low carbon energy solutions for remote rural areas of the developing world.

The primary objectives of the study were (a) to propose a low cost, low emissions, renewable alternative cooking fuel to charcoal for the remote rural population of Niger State, (b) to embed low carbon technologies to the proposed process's associated heat and power demand, (c) to provide process design characteristics variable scale for community use, and (d) to determine associated costs by making use of local materials and low maintenance requirements adjusted to local population constraints.

\section{Experimental method}

\subsection{Selection of technology according to landscape and scale}

The viability of a given technology in a given area is dependent upon certain factors; suitability of scale, availability of feedstock/input, geography and geology of the area, average sunlight/winds speed/rainfall. All of these factors were considered approaching the design in order to provide a flexible and reliable energy solution for as broader range of situations as possible. A simplified yes/no method has been created to determine the capability of a technique suitable to Niger State's energy needs. Furthermore the scalability of each technology, from domestic to regional use, determines its potential in rural and remote areas. For both tables a legend has been created for ease of understanding and 
uniformity throughout. A tick merely defines the likely probability of this technology working for the suggested situation, whilst a cross is an unlikely or impractical use. A question mark defines a situation information is lacking so a definite yes/no decision cannot be made; or the use of this technology is situational, such as seasonal changes, economics or no examples currently exist.

Table 1. Applicability of each representative technology in a landscape.

\begin{tabular}{|l|c|c|c|c|c|c|}
\hline & Hydro & Wind & AD & Solar & Tidal & Geother \\
\hline Flood Plains & $\checkmark$ & $\checkmark$ & $\checkmark$ & $\checkmark$ & $x$ & $?$ \\
\hline Plains & $x$ & $\checkmark$ & $\checkmark$ & $\checkmark$ & $x$ & $?$ \\
\hline Hills & $\checkmark$ & $\checkmark$ & $\checkmark$ & $\checkmark$ & $x$ & $?$ \\
\hline Mountains & $\checkmark$ & $\checkmark$ & $\checkmark$ & $\checkmark$ & $x$ & $?$ \\
\hline Tundra & $\checkmark$ & $\checkmark$ & $x$ & $\checkmark$ & $x$ & $?$ \\
\hline Dessert (HOT) & $x$ & $?$ & $?$ & $\checkmark$ & $x$ & $?$ \\
\hline Dessert (COLD) & $x$ & $\checkmark$ & $x$ & $\checkmark$ & $x$ & $?$ \\
\hline Savanah & $\checkmark$ & $?$ & $\checkmark$ & $\checkmark$ & $x$ & $?$ \\
\hline Jungle & $\checkmark$ & $x$ & $\checkmark$ & $x$ & $x$ & $?$ \\
\hline Forest & $\checkmark$ & $x$ & $\checkmark$ & $x$ & $x$ & $?$ \\
\hline Marsh/wetlands & $?$ & $?$ & $\checkmark$ & $\checkmark$ & $x$ & $?$ \\
\hline Delta/River & $\checkmark$ & $\checkmark$ & $\checkmark$ & $\checkmark$ & $\checkmark$ & $?$ \\
\hline Coastline & $\checkmark$ & $\checkmark$ & $\checkmark$ & $\checkmark$ & $\checkmark$ & $?$ \\
\hline Atoll & $x$ & $\checkmark$ & $\checkmark$ & $\checkmark$ & $\checkmark$ & $?$ \\
\hline Volcanic & $x$ & $\checkmark$ & $?$ & $\checkmark$ & $x$ & $?$ \\
\hline
\end{tabular}

Table 2. Applicability and scalability of each representative technology.

\begin{tabular}{|c|c|c|c|c|c|c|c|}
\hline \multirow{2}{*}{\multicolumn{2}{|c|}{$\begin{array}{c}\text { Applicable use of each } \\
\text { technology based upon } \\
\text { home powerage }\end{array}$}} & \multicolumn{6}{|c|}{ Scale } \\
\hline & & Household & $\begin{array}{c}\text { Multiple } \\
\text { House }\end{array}$ & Community & $\begin{array}{c}\text { Multiple } \\
\text { community }\end{array}$ & $\begin{array}{l}\text { Urban } \\
\text { centre }\end{array}$ & Regional \\
\hline \multirow{12}{*}{ Tech } & Pico Hydro & $\checkmark$ & $\times$ & $x$ & $x$ & $x$ & $x$ \\
\hline & Micro Hydro & $\checkmark$ & $\checkmark$ & $\checkmark$ & $x$ & $x$ & $x$ \\
\hline & Small Hydro & $x$ & $\times$ & $\checkmark$ & $\checkmark$ & $x$ & $x$ \\
\hline & Hydro $(+10 \mathrm{MW})$ & $x$ & $x$ & $x$ & $x$ & $\checkmark$ & $\checkmark$ \\
\hline & Tidal & ? & $\checkmark$ & $\checkmark$ & $\checkmark$ & $\checkmark$ & $\checkmark$ \\
\hline & Solar & $\checkmark$ & $\checkmark$ & $\checkmark$ & $\checkmark$ & $\checkmark$ & $\checkmark$ \\
\hline & Solar Tower* & $x$ & $x$ & $x$ & $x$ & $\checkmark$ & $\checkmark$ \\
\hline & Small wind & $\checkmark$ & $\checkmark$ & $x$ & $x$ & $\boldsymbol{x}$ & $x$ \\
\hline & Wind & $\checkmark$ & $\checkmark$ & $\checkmark$ & $\checkmark$ & $\checkmark$ & $\checkmark$ \\
\hline & $A D$ & $\checkmark$ & $\checkmark$ & $\checkmark$ & $x$ & $\checkmark$ & $\checkmark$ \\
\hline & Biofuels & $x$ & $\times$ & $\mathbf{x}$ & $x$ & $x$ & $\checkmark$ \\
\hline & MSW & $x$ & $x$ & $x$ & $x$ & $\checkmark$ & $\checkmark$ \\
\hline
\end{tabular}

\subsection{Quality function deployment}

Quality function deployment (QFD) is a tool used to relate customer requirements to the technical aspects of the design. There are four main aspects to the QFD. The customer requirements are shown on the left hand side of the house of quality (HOQ). This is a list of all that the customer requires from the design e.g. clean biogas. The customer requirements often are produced through interviews, however the customer requirements in this study have been discovered through literature. The technical requirements are listed along the top of the HOQ. The technical requirements should be measureable and should be solutions to the customer's requirements e.g. size and volume. An additional row is added to show if the technical requirement needs to be maximized or minimized. The main block of the house contains the inter-relationships between the customer requirements and technical requirements. The relationships are described as either direct, strong, weak or indirect.

In out QFD diagram (available as supplementary data), these relationships have been colour coded to make for ease of viewing. The roof of the HOQ allows links to be formed between the technical aspects of the design. The question is asked 'how does one requirement 
affect the performance of another?' The relationship maybe positive such as the relationship between corrosion resistance and material lifetimes. Negative such as the relationship between simplicity and the size and volume of the system, or no relationship such as the relationship between materials used and the plant location.

\subsection{Objectives tree}

The objectives tree is the third technique used in the present study to produce the best low carbon energy system for Niger State's rural population's alternative cooking fuel. Starting with the top level requirement of 'Integrated rural energy solution: community/domestic scale anaerobic digestion - solar PVT/thermal system', the tree is split into functional requirements and operational improvements. The functional requirements are highlighted in red and the operational requirements are blue, again to make for ease of viewing (see supplementary data). The functional requirements are then spilt into providing a range of solutions to meet the requirement e.g. mesophilic or thermophilic for a microorganism population. The operational improvements are however more specific and are more of an aim e.g. consistent temperature. Within the objectives tree the shape of the design begins to take place, allowing ideas to flow about what needs to be included in the design to meet customer needs. The importance of a simple design became incredibly apparent during the objectives tree. A simple design leads to simple maintenance, repair and installation.

\section{Results and Discussion}

Using the methodologies described in 2 to the geographical area of Niger State (Savanah and flood-planes, Table 2), and to small community application representative of remote agricultural populations (Table 3), two energy resources stand out as suitable low carbon energy solutions: anaerobic digestion and solar. Added too these the outcomes of the QFD and Objective tree, the proposed solution to the low carbon energy challenge within Niger State's provision of alternative cooking fuel converged to proposing biogas generated by an integrated solar Photovoltaic-Thermal (PVT) and Anaerobic Digestion (AD) unit, using mesophilic technology for the community scale. The AD system was characterised by a fixed volume corresponding to communities of up to 15 households, an agitator powered by the electricity from the solar PVT, a heating system provided by the solar PVT, an inlet and outlet, mesh in order to keep floating biomass submerged, thermometer, entrance hatch and a $\mathrm{H}_{2} \mathrm{~S}$ scrubber. Community scale AD plant (ADP) cost is fundamentally dependent upon integration of solar PVT and volumetric capacity. Total biogas production is predominantly affected by feedstock type and availability.

\subsection{Design of a community anaerobic digester}

The AD tank is $20 \mathrm{~m}^{3}$ in volume, it is designed to have flat walls yet a rounded bottom with no sharp corners to minimize sludge accumulation in corners. The AD tank is to be made from bricks and cement with a polyethylene (PE) lining. The materials are widely available as well as being economically affordable. The materials provide the best solution regarding simplicity, economically affordability and longevity of the system.

The digestion medium will be agitated in order to increase the efficiency of the AD system. The medium is also held down by a mesh to prevent floating waste from not being digested. The agitator will be powered by the electricity produced by solar PVT and should therefore not require any external electricity to run.

The solar PVT is also providing hot water to the anaerobic digester. Through a series of copper pipes hot water will be distributed throughout the system. The hot copper pipes will 
aim to keep the medium at $37^{\circ} \mathrm{C}$, and thus running at optimum efficiency. The temperature of the digester will be monitored by a thermometer displayed at the top of the system.

\subsubsection{Material requirements}

Typically AD reactors, in an attempt to improve deployability and reduce cost, utilize poured concrete, brick and stone materials often resulting in enhanced porosity and gas-loss over time [11]. In addition, fixed-dome designs may incorporate reinforced-concrete, fiberglass-reinforced-polyester and steel with additional internal mortar layers applied so as to maintain anaerobic-conditions within the ADP [11]. Concrete is applied due to ease of application, capacity for air-retention and water-retention, and resistance to thermal inertia [12].

Traditionally, alkaline concrete is directly exposed to the solid-liquid phase of the digestion medium resulting in corrosive deterioration through biogenic sulphuric-acid generation [13]. Corrosive decline of the structure may be maintained at $1 \mathrm{~mm} /$ year [13], dependent upon internal and operational conditions. In addition, volatile fatty acids and ammonia accumulation within the solid/liquid phase may contribute in "concrete leeching" [13] with dissolved- $\mathrm{CO}_{2}$ generation [11] may be considered a source of concrete carbonization [12]. Such processes result in reduced efficiency and costly repair-works with risk of gas liberation and solid/liquid discharge within the adjacent environment [11].

\subsubsection{Biogas scrubbing}

Of all the impurities commonly present in biogas $\mathrm{H}_{2} \mathrm{~S}$ is the most pressing when used as a energy solution in this fashion, used as an alternative cooking fuel it would be damaging to health and co-ignited in a diesel engine it would cause rapid engine wear.

$\mathrm{H}_{2} \mathrm{~S}$ remediation may be achieved through conversion to sulphurous-acid via passage between a solid-filter-pack of iron-wool, the transfer conversion of iron-wool to FerricSulphide (FeS) negates the presence of detrimental $\mathrm{H}_{2} \mathrm{~S}$ within the biogas-compound [14]. In addition, limitations in iron-wool surface area inhibits conversion potential, as such low-cost wood-chippings with iron-oxide $\left(\mathrm{Fe}_{2} \mathrm{O}_{3}\right)$ may be applied so as to enhance surface-area ratios [14]. Approximately $20 \mathrm{~g}$ of $\mathrm{H}_{2} \mathrm{~S}$ may be remediated per $100 \mathrm{~g}$ of wood-chipping iron-oxide filter-pack [15].

\subsubsection{Agitation}

The application of mixing is established as a method of improved operational performance within ADP's [16]. Advantageous performance is achieved through augmented nutrientdistribution and digestion medium inoculation; uniformity of heating; negation of scum and foam formation; biogas bubble release and disturbance of feedstock sedimentation [17]. Rates of mixing tend to be low, usually within the range of 15-50 rpm, a mechanical propeller type stirrer is identified for universal application [17].

\subsubsection{Shape and size}

ADP operational size is determined as a function of feedstock volume availability $(\mathrm{kg})$, biogas potential $\left(\mathrm{m}^{3} / \mathrm{kg}\right)$ and consumption demand $\left(\mathrm{m}^{3} / \mathrm{kg}\right)$ specific to the location of deployment [18,19]. Attainment of ADP size adequacy should be conceived with consideration for capital and labour expense associated with excessive construct volume and material investment [18]. In addition, variability in the presumed feedstock capacity for biogas generation [20] should be considered when assessing the volumetric dimensions of presumed systems [19]. A linear relationship may be assumed between feedstock mass, supportable population and integrated ADP volume $\left(\mathrm{m}^{3}\right)$ [18]. In addition, ADP size 
maintains a direct impact upon land-use patterns, fresh-water investment and heating intensity.

A numerical estimation of consumer population (P), per ADP device, is determined a prerequisite when considering total biogas demand and operational plant size [19]. The total daily-biogas requirement $\left(\mathrm{G}_{\mathrm{r}}\right)$ per consumer population (Eq.1) is initially considered in volumetric ADP design.

Notable variation in average household size is also presented with the greatest proportion of people living within 8 person households $(F)$ across Niger State [21]. Cooking requirements per inhabitant $(C)$ are estimated to be $0.227 \mathrm{~m}^{3}[22,23]$ with lighting requirement per household $(L)$ within the region of $0.125 \mathrm{~m}^{3}$ per gas-lamp per hour $\left(L_{1} \cdot L_{2}\right)$ [22]. Assuming lighting requirements of 2 lighting hours per day the total estimated biogas demand is $2.09 \mathrm{~m}^{3}$. The estimation of $2 \mathrm{~m}^{3}$ biogas demand is equivalent to $1805.11 \mathrm{~kg} /$ year and $722.05 \mathrm{~kg} / \mathrm{year}$ of firewood and charcoal respectively [22].

A total biogas potential (A) is dependent upon both number of livestock $(z)$, mass of feedstock production per day $(\mathrm{kg})\left(\mathrm{z}_{1}\right)$, and biogas potential per unit of substrate $\left(\mathrm{m}^{3} / \mathrm{kg}\right)(x)$ $[22,23]$. A total of 7 head of cattle per household, a dung production rate of $10 \mathrm{~kg}$ per day [22] and biogas potential of $0.03 \mathrm{~m}^{3} / \mathrm{kg}$ is (equation 1). As such a total biogas potential of $2.10 \mathrm{~m}^{3}$ is estimated for this case study.

$$
G_{r}=\left[(\mathrm{P} \times \mathrm{C})+\left(\mathrm{L} \times L_{1} \times L_{2}\right)\right]
$$

An estimation of digester liquid phase volume $\left(V_{d}\right)$ (equations $2 \& 3$ ) is calculated for dry matter feedstock proportion $(M)$ of $10 \%$ [24], a dry feedstock density $(\Psi)$ of $50 \mathrm{~kg} / \mathrm{m}^{3}$ [22] and a hydraulic retention time $\left(t_{r}\right)$ of 30 days [22]. As such a total digester volume of $4.18 \mathrm{~m}^{3}$ would be required for the liquid phase proportion of the ADP structure. An additional 10\% volume size is determined for gas generation disengagement over time [22] suggesting a minimum ADP volumetric capacity of $4.598 \mathrm{~m}^{3}$.

$$
\begin{array}{r}
\mathrm{A}=\left(z \times z_{1} \times x\right) \\
V_{d}=\frac{\left[(\mathrm{D} \times \mathrm{C})+\frac{\left(\mathrm{L} \times L_{1} \times L_{2}\right)}{x}\right]}{\Psi} \times t_{r}
\end{array}
$$

The proposed set up of the integrated solution is shown above in Figure 1, the biogas scrubbing unit, which on the diagram is on the reactor but would be a small and mobile unit. The submerged piping serves to distribute heat from the solar PVT around the reactor.

\subsubsection{PVT specifications}

The specifications of the solar PVT system associated with the chosen design are defined here to determine the required coverage according to reactor size. Reactor size represents the rough estimate of the water content of the reactor; Coverage required refers to the area to be covered with the PVT panels and is determined using Equation 4. The required temperature is lower than ideal because a $1^{\circ} \mathrm{C}$ rise leads to a $50 \%$ increase in required coverage.

$$
\mathrm{CR}=(\mathrm{RT}-\mathrm{AT}) * \operatorname{Erq} *(\mathrm{RS} * 1000)
$$

Parameters in Eq. 4 are defined in Table 3, along with their values evaluated for the chosen design. 


\subsection{Estimating Initial Capital Investment for Community Scale Anaerobic Digester}

Significant capital cost $(\mathrm{Cc})$, associated with large-scale systems, is identified as a characteristic inhibiting ADP deployment. However, in terms of energy production capacity, ADP systems present the most cost effective renewable energy resource, an aspect of paramount importance in developing nations. Enhancement in operational volume is synonymous with increase in capital-cost. As previously suggested, volume requirement is the result of biogas potential, feedstock supply, density and hydraulic retention time. The development of community-scale ADP, and associated capital-cost, is therefore dependent upon feedstock typology and availability, biogas demand and hydraulic retention time.

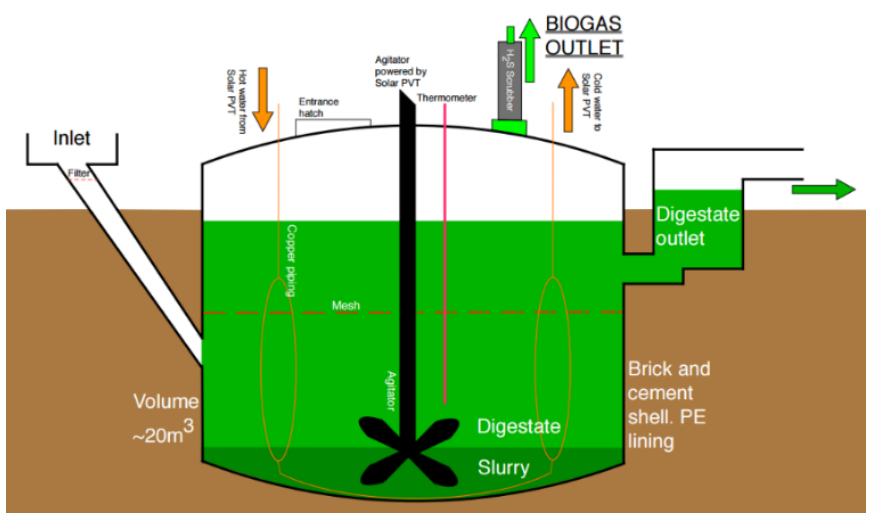

Fig. 1. Community scale design for an AD plant using solar thermal.

Table 3. Specifications of the solar PVT system.

\begin{tabular}{|c|c|c|c|}
\hline Energy to raise 1 litre of water by $\mathbf{1}^{\circ} \mathbf{C}$ & $\mathbf{0 . 0 0 1 1 6 1}$ & $\mathbf{k W h}$ & Erq \\
\hline Reactor Size & 15 & $\mathrm{~m}$ & $\mathrm{RS}$ \\
\hline Annual average temp (2015) & 27.4 & ${ }^{\circ} \mathrm{C}$ & $\mathrm{AT}$ \\
\hline Required temp & 29 & ${ }^{\circ} \mathrm{C}$ & $\mathrm{RT}$ \\
\hline Energy required & 27.9 & $\mathrm{kWh}$ & \\
\hline Nigeria average solar radiation & 7 & $\mathrm{kWh} / \mathrm{m}^{2}$ & \\
\hline Expected eff. of PVT & 0.45 & & \\
\hline Daily generation per $\mathrm{m}^{2}$ & 3.15 & $\mathrm{kWh}$ & \\
\hline Coverage required & 8.8 & $\mathrm{~m}^{2}$ & $\mathrm{CR}$ \\
\hline
\end{tabular}

An estimation of the volumetric ADP requirement was established for varying numbers of community consumers (Equations 2 and 3). An estimated cost formula was developed integrating fundamental requirement and material volume for an ADP system of varying size. Results indicate variation in initial capital investment depending upon integration of solar PVT and volume of ADP digester. Estimations suggest a community of 24 people would require a reactor size of $12.7 \mathrm{~m}^{3}$ at a cost of 4613.77 US\$ when PVT is integrated. Removing the PTV system significantly reduces the cost to 1338.79 US\$ for a system of equal volume. In addition, the total initial capital investment is shown to reduce, in proportion to ADP volume, with an increase in size. 


\section{Conclusion}

The proposed solution to provision of clean alternative cooking fuel to solid biomass for remote communities in Niger State is biogas generated by anaerobic digestion mesophilic technology with digester temperature control as well as agitator power provided by hybrid solar PV-T panel. The system also features a design allowing flexible range of digester volume, an inlet and outlet, an internal mesh keeping floating biomass submerged, a thermometer, an entrance hatch and a wood chipping iron oxide based $\mathrm{H}_{2} \mathrm{~S}$ scrubber, and is made of local construction materials. Total biogas production is predominantly affected by feedstock type and availability. The solution has potential to save lives and increase life expectancy by reducing the amount of harmful gases inhaled by cooking with poor quality fuels in inefficient cook stoves.

\section{Acknowledgments}

The EPSRC is gratefully acknowledged for GCRF grant EP/P51097X/1 (Integrated Low Carbon Energy solutions for Remote Rural Area project) and EP/L014912/1 (CDT bioenergy) programme at The University of Leeds. Supplementary data available at http://eprints.whiterose.ac.uk/ under title name of paper.

\section{References}

1. United Nations, Department of Economic and Social Affairs. Population division. (2015)

2. A.C. Ohajianya, O.E. Abumere, I.O. Owate, E. Osarolube, Int. J. Eng. Sci. Inv. 3, 7 (2014)

3. IEA. Nigeria: Balances for 2014 (2014)

4. The World Bank. Population Total (2016)

5. I.U. Abubakar, M.A. Yamusa, Int. J. Agri. Food. Sci. Tech. 4, 3 (2013)

6. O.A. Olayo, J. Food. Sec. 2, 1 (2014)

7. V.O. Oladokun, O.C. Asemota, Renew. Sust. Energy. Rev. 52 (2015)

8. K. Ley, J. Gaines, A. Ghatikar, GmbH (GIZ) (2014)

9. IEA, Statistics (2016)

10. IEA, National Energy Policy (2016)

11. C. Gunnerson, D. Stuckey, World Bank Technical Paper 49 (1986)

12. C. Voegel, A. Bertron, B. Erable, Sci. Total. Environ. 571 (2016)

13. A. Koenig, F. Dehn, Biosys. Eng. 147 (2016)

14. A. Agarwal, M. Shukla, Int. J. Oil. Gas. Coal. T. 2, 4 (2009)

15. K. Krich, D. Augenstein, J. Batmale, J. Benemann, B. Rutledge, D. Salour, Clean Concepts (2005)

16. D. Stafford, Biomass 2, 1 (1982)

17. A. Lemmer, H. Naegele, J. Songermann, Energies 6, (2013)

18. K. Singh, S. Sooch, Energ. Convers. Manage. 45, 9-10 (2004)

19. S. Sakar, K. Yetilmezsoy, E. Kocak, Waste. Manage. Res. 27 (2009)

20. L. Makai, M. Molinas, GHTC (2013)

21. National Population Commission (Nigeria) (2006) 
22. G. Rai, Non-Conventional Energy Resources (New Delhi, 2002)

23. E. Alokore, B. Ebangu, Agri. Eng. Int. 15, 1 (2013)

24. T. Pullen, Anaerobic Digestion, Making Biogas, Making Energy (The Earthscan Expert Guide, Routledge, 2015) 